\section{Körperliche (In-)Aktivität und Schwere der COVID-19 Verläufe}

Die negativen gesundheitlichen Auswirkungen körperlicher Inaktivität sind unbestritten. Nicht nur erhöht sich mit steigender Inaktivität die Erkrankungswahrscheinlichkeit nicht übertragbarer chronischer Erkrankungen wie Diabetes mellitus Typ 2 [1], Bluthochdruck [2], Osteoporose [3, 4] oder Krebs [5], körperliche Inaktivität ist auch mit einer erhöhten Sterblichkeit assoziiert [6]. In Anbetracht der hohen Aktualität stellt sich daher unmittelbar die Frage, wie sich körperliche Aktivität auf die Schwere und Mortalität von COVID-19 auswirkt.

In einer spanischen Kohorte [7] wurden im Zeitraum von Februar bis April 2020 insgesamt 556 COVID-19 Patienten registriert. Nach Entlassung aus dem Krankenhaus wurden die Patienten kontaktiert, um Auskunft über ihr Aktivitätsverhalten zu geben. War ein Patient verstorben, wurde ein Familienangehöriger kontaktiert und um eine Einschätzung gebeten. Entsprechend des berichteten Aktivitätsverhaltens wurden die Patienten in zwei Gruppen eingeteilt. Der ersten Gruppe wurden jene Menschen zugeordnet, welche vornehmlich einen sitzenden Lebensstil pflegten und wenig aktiv waren, der zweiten Gruppe wurden jene zugeordnet, die regelmäßig körperlich aktiv waren. Beim Vergleich der Gruppenmerkmale fielen substanzielle Unterschiede zwischen der aktiven und inaktiven Gruppe auf. Beispielswiese wiesen die Aktiven weniger Komorbiditäten und einen tendenziell gesünderen Lebensstil auf. Im Hinblick auf die COVID-19 assoziierte Mortalität konnte in der inaktiven Gruppe eine 8,13 (95\% Cl 2,91-22,70) -fach erhöhte Sterblichkeit verglichen mit der aktiven Gruppe beobachtet werden. Hiermit hob sich die Gruppenzuweisung als wichtigster Mortalitätsprädiktor hervor und blieb auch nach Adjustierung für verschiedene Covariaten substanziell mit einer Hazard-Ratio von 5,91 (1,80-19,31) substanziell erhöht.

In einer weiteren, in Brasilien durchgeführten ökologischen Beobachtungsstudie [8], also einer Studie, in welcher nicht die Merkmale einzelner Personen, sondern jene von ganzen Regionen verglichen oder in Zusam- menhang gesetzt werden, wurden die COVID-19 Mortalitätsraten mit dem Anteil der Bevölkerung in Beziehung gesetzt, welche körperlichen Freizeitaktivitäten nachgeht. Hierzu lagen Daten von allen 26 brasilianischen Bundesstaaten und ihren Hauptstädten vor. Via multipler Regressionsanalyse, in welcher für Übergewicht und Bluthochdruck adjustiert wurde, konnten die Autoren eine Reduktion der COVID-19 Letalität, also der Versterbewahrscheinlichkeit bei vorliegender COVID-19 Diagnose, beobachten.

Die bislang methodisch hochwertigste und belastbarste Arbeit wurde im British Journal of Sports Medicine publiziert und betrachtet die Daten von fast 50.000 erwachsenen Patienten aus Kalifornien, welche zwischen Januar und Oktober 2020 eine COVID-19 Diagnose gestellt bekamen [9]. Von den eingeschlossenen Patienten lagen zum Zeitpunkt der Diagnose bereits mindestens drei Angaben hinsichtlich des Aktivitätsniveau aus den vorangegangenen Jahren vor. Die eingeschlossenen Patienten wurden drei Gruppen zugeordnet. Jene, welche die WHO Guidelines von über 150 min moderater bis intensiver körperlicher Aktivität pro Woche (moderate to vigerous physical activity MVPA) erreichten, bildeten die Referenzgruppe. Mäßige Aktive wurden mit einem Aktivitätsumfang von 11-149 min MVPA pro Woche definiert und machten die zweite Gruppe aus. Die letzte Gruppe waren die inaktiven Personen, die weniger als $10 \mathrm{~min} /$ Woche moderat bis intensiv aktiv waren. Hinsichtlich der Gruppencharakteristika fallen, ebenso wie in der spanischen Kohorte oben, einige substanzielle Unterschiede auf. Beispielsweise war die aktive Referenzgruppe im Mittel neun Jahre jünger als die inaktive Gruppe. Insbesondere der Anteil betagter Menschen lag mit einem in fünf wesentlich höher als in der aktiven Gruppe mit lediglich 1,4\%. Auch hinsichtlich der Ethnie gab es in den verschiedenen Gruppen deutliche Unterschiede. Insgesamt bestand die Gesamtstichprobe zu etwa zwei Dritteln aus Hispanoamerikanern. Des Weiteren zeigte die inaktive Gruppe weitere gesundheitliche Risikofaktoren, wie einen höheren Anteil von Rauchern, einen höheren BMI sowie verschiedene Komorbiditäten, welche gleichzeitig auch mit dem höheren Alter dieser Gruppe assoziiert sind.
Als Endpunkte wurden Hospitalisierung, Behandlung auf Intensivstation und die Mortalität definiert. In der gesamten Kohorte wurden 4236 (8,7\%) hospitalisiert, 1199 (2,5\%) bedurften Behandlung auf der Intensivstation und 771 (1,6\%) verstarben. In allen drei Endpunkten zeigte sich ein positiver Zusammenhang zur körperlichen Inaktivität. Auch nach Adjustierung für die oben angeführten Kovariaten blieb der Effekt erhalten. Konkret zeigte sich hinsichtlich der inaktiven Gruppe ein 2,26-faches Hospitalisationsrisiko (95\% Cl 1,81-2,83), ein 1,73-faches Risiko (95\% Cl 1,18-2,55) eines intensivstationären Behandlungsbedarfs sowie eine 2,49-fache ( $95 \% \mathrm{Cl} 1,33$ $4,67)$ Mortalität. Die mäßig Aktiven lagen in allen drei Outcomes zwischen der aktiven und inaktiven Gruppe. Sie zeigten ein 1,89-faches Hospitalisationsrisiko (1,532,33), ein 1,58-faches Risiko der Betreuung auf der Intensivstation $(1,10-2,27)$ und eine 1,88 -fache Mortalität $(1,02-3,47)$ als sie in der aktiven Referenzgruppe zu beobachten war. Hierbei ist auffällig, dass sich die Referenzgruppe in allen Outcomes signifikant von den beiden anderen Gruppen abhebt, die Unterschiede der beiden anderen Gruppen das statistische Signifikanzniveau allerdings nicht erreichen. Da sich in allen drei Endpunkten allerdings ein ähnlicher Trend abzeichnet, kann ein Dosis-Wirkungs-Zusammenhang mit gutem Gewissen vermutet werden.

Die vorgestellten Arbeiten zeigen, dass das körperliche (In-)Aktivitätsniveau vor Diagnosestellung ein wirkmächtiger Prädiktor im Hinblick auf die Schwere des COVID-19 Verlaufs darstellt. Da es sich in allen drei Fällen jedoch um Beobachtungsstudien handelt, wäre der Schluss auf einen Kausalzusammenhang zu voreilig. Es wäre ebenso denkbar, dass die starke Assoziation zwischen körperlicher (In-)Aktivität und Mortalität durch einen nicht erhobene Kovariate mediiert wird. Schließlich lagen im Hinblick auf Lebensstilfaktoren und Komorbiditäten bereits substanzielle Unterschiede zwischen den Gruppen vor. Ein weiteres zentrales Manko ist, wie in so gut wie allen bewegungsepidemiologischen Studien, die Erhebung der körperlichen Aktivität. Diese erfolgte subjektiv durch Selbstbericht. Hinzu kommt, dass weder Typ noch Intensität der Aktivitäten ausreichend erhoben wurden. Im Fall der Arbeit 
von Salgado-Aranda und Kollegen ist außerdem hervorzuheben, dass die Patienten retrospektiv nach ihrem Aktivitätsverhalten gefragt wurden, wodurch die Verlässlichkeit der berichteten Werte zusätzlich verzerrt sein kann. Dennoch liegen nachvollziehbare Erklärungsansätze für den beobachteten protektiven Effekt körperlicher Aktivität vor. So spekulieren Salgado-Aranda et al., dass dieser Effekt insbesondere auf die höhere Sauerstoffaufnahmekapazität aktiver Menschen zurückzuführen ist. Konkret zeigte sich in der aktiven Gruppe eine höhere Sauerstoffsättigung sowie selteneres Lungenversagen trotz vergleichbarer radiologisch abgesicherter Pathologie.

Auch im Hinblick auf COVID-19 scheint körperliche Aktivität eine wichtige Gesundheitsressource darzustellen. Die weltweit zu beobachtenden massiven Aktivitätseinbrüche von im Mittel 41 \% [10], welche während der Pandemie zu beobachten sind, erscheinen in diesem Kontext umso beunruhigender und bedürfen in naher Zukunft einer angemessenen Adressierung.

\section{Korrespondenzadresse}

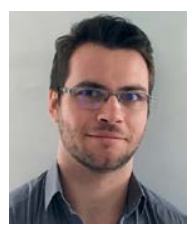

Maximilian Köppel

Deutscher Verband für Gesundheitssport und Sporttherapie e.V. Vogelsanger Weg 48 50354 Hürth-Efferen Deutschland Maximilian.koeppel@outlook.de
Literature

[1] Lindström J, Peltonen M, Eriksson J et al. Improved lifestyle and decreased diabetes risk over 13 years: long-term follow-up of the randomised Finnish Diabetes Prevention Study (DPS). Diabetologia 2013; 56: 284-293

[2] Börjesson M, Onerup A, Lundqvist S, Dahlöf B. Physical activity and exercise lower blood pressure in individuals with hypertension: narrative review of 27 RCTs. British journal of sports medicine 2016; 50: 356-361

[3] Langsetmo L, Hitchcock C, Kingwell E et al. Physical activity, body mass index and bone mineral density-associations in a prospective population-based cohort of women and men: The Canadian Multicentre Osteoporosis Study (CaMos). Bone 2012; 50: 401-408

[4] Pinheiro MB, Oliveira J, Bauman A et al. Evidence on physical activity and osteoporosis prevention for people aged 65 + years: a systematic review to inform the WHO guidelines on physical activity and sedentary behaviour. International Journal of Behavioral Nutrition and Physical Activity 2020; 17: 1-53

[5] Patel AV, Friedenreich CM, Moore SC et al. American College of Sports Medicine roundtable report on physical activity, sedentary behavior, and cancer prevention and control. Medicine and science in sports and exercise 2019; 51: 2391
[6] Lee I-M, Shiroma E], Lobelo F et al. Effect of physical inactivity on major non-communicable diseases worldwide: an analysis of burden of disease and life expectancy. The lancet 2012; 380: 219-229

[7] Salgado-Aranda R, Pérez-Castellano N, Núñez-Gil I et al. Influence of Baseline Physical Activity as a Modifying Factor on COVID-19 Mortality: A Single-Center, Retrospective Study. Infectious Diseases and Therapy 2021; 10: 801-814

[8] Pitanga FJG, Beck CC, Pitanga CPS et al. Association between leisure time physical activity and mortality by COVID-19 in the Brazilian Capitals: An ecological analysis. 2021;

[9] Sallis R, Young DR, Tartof SY et al. Physical inactivity is associated with a higher risk for severe COVID-19 outcomes: a study in 48 440 adult patients. British journal of sports medicine. 2021;

[10] Tison GH, Avram R, Kuhar P et al. Worldwide effect of COVID-19 on physical activity: a descriptive study. Annals of internal medicine 2020; 173: 767-770 